\title{
Status and manifestations of sleep disorders and the status of drugs used to help fall sleep in Chinese breast cancer women at initial diagnosis and during chemotherapy
}

\section{Baoshan Zou}

First Affiliated Hospital of Chongqing Medical University

Hong Li

First Affiliated Hospital of Chongqing Medical University

Jiashuo Liu

First Affiliated Hospital of Chongqing Medical University

Zhou Xu

First Affiliated Hospital of Chongqing Medical University

Haoran Chen

First Affiliated Hospital of Chongqing Medical University

Hao Li

First Affiliated Hospital of Chongqing Medical University

Kainan Wu

First Affiliated Hospital of Chongqing Medical University

Lingquan Kong ( $\nabla$ huihuikp@163.com )

First Affiliated Hospital of Chongqing Medical University

https://orcid.org/0000-0001-5705-9001

\section{Research article}

Keywords: breast cancer, sleep disorders, chemotherapy, the Pittsburgh Sleep Quality Index

Posted Date: October 9th, 2019

DOI: https://doi.org/10.21203/rs.2.15785/v1

License: @ (i) This work is licensed under a Creative Commons Attribution 4.0 International License. Read Full License 


\section{Abstract}

Purpose This study was designed to investigate the status and manifestations of sleep disorders and the status of drugs used to help fall sleep in Chinese breast cancer women at initial diagnosis and during chemotherapy.

Patients and Methods Totally 308 primary breast cancer women were enrolled. The status and manifestations of sleep disorders and the status of drugs used to help fall sleep in the above breast cancer patients were assessed via the Pittsburgh Sleep Quality Index (PSQI).

Result The prevalence of sleep disorders in patients at the time of initial diagnosis with breast cancer was approximately $50 \%(97 / 194)$, which was significantly lower than that $(65.8 \%, 75 / 114)$ in breast cancer patients before the last cycle of chemotherapy $(\mathrm{p} \otimes 0.05)$. However, both groups of patients rarely used drugs to help them fall sleep ( $P>0.05)$. The mean scores for sleep latency, habitual sleep efficiency, sleep disturbance, and daytime dysfunction $(1.68,1.08,1.65,1.37)$ in breast cancer patients prior to the last cycle of chemotherapy were obviously worse than those $(1.29,0.71,1.26,1.01)$ in breast cancer patients at the time of initial diagnosis $(p<0.05)$.

Conclusion Chemotherapy mainly affects the sleep latency, habitual sleep efficiency, sleep disturbance, daytime dysfunction in breast cancer patients. Chinese breast cancer patients rarely use drugs to intervene sleep disorders.

\section{Introduction}

In the past few decades, the trends towards the earlier detection of breast cancer and improvements in breast cancer treatment have increased the incidence and improved survival ${ }^{[1-3]}$. Currently, breast cancer is one of the most common diseases, with an estimated 1.7 million new cases in 2012, accounting for $25 \%$ of all cancers in women[4]. The incidence of sleep disorders varies based on population characteristics, breast cancer stages, definitions of sleep disorders and different evaluation criteria, but it is clear that a large number of patients with breast cancer have sleep disorders ${ }^{[5]}$. Most studies report that $30 \%$ to $50 \%$ of cancer patients have sleep disorders at the time of their initial diagnosis, and the prevalence of sleep disorders is the highest in patients with breast and gynecologic cancers ${ }^{[6-8]}$. The proportion of breast cancer patients with sleep disorders is higher than that observed in healthy adults ${ }^{[9]}$. Insomnia is the most common sleep disorder. A study has shown that breast cancer patients have a three-fold higher incidence of insomnia than the general population ${ }^{[10,11]}$. Most of the symptoms of insomnia are subjective complaints of difficulty sleeping. The symptoms mainly include difficulty falling asleep, difficulty staying sleeping, early waking, and unstable or poor sleep quality.

All adjuvant treatments for breast cancer patients have an impact on sleep quality because of their psychological and physiological effects and side effects. Chemotherapy is thought to be particularly harmful $^{[12,13]}$. Breast cancer patients have more subjective sleep disorders during chemotherapy than 
during non-treatment periods ${ }^{[14,15]}$. Longitudinal studies have also shown that the quality of sleep in breast cancer patients continues to decline during the chemotherapy cycle $\mathrm{e}^{[16]}$.

The Pittsburgh Sleep Quality Index (PSQI) has been shown to be an accurate psychological tool that can be used to directly screen for sleep disorders in cancer patients[17]. The PSQI is a 19-item self-reporting tool used to assess sleep disorders in the month prior to the administration of the questionnaire. Seven component scores are calculated from the questionnaire: subjective sleep quality, sleep latency, sleep duration, habitual sleep efficiency, sleep disturbance, sleep medications, and daytime dysfunction. Sleep disturbance is defined as poor sleeping, including difficulty falling asleep, bathroom use, breathing difficulty, waking up at night, coughing, snoring, dreams, pain and uncomfortable temperature. The scores for these components are ranked from 0 (no difficulty) to 3 (severe difficulty) and are summed to produce a global measurement of sleep disorders. The global score ranges from 0 to 21 ; the higher the score is, the worse the quality of sleep. A total score above 5 is generally defined as the presence of a sleep disorder[18].

There are few studies about the manifestations of sleep disorders as measured by the PSQI and the status of the drugs used to help Chinese breast cancer patients fall asleep. Therefore, this study was designed to investigate the status and manifestations of sleep disorders, as measured by the PSQI, and the status of the drugs used to help Chinese women with breast cancer fall asleep at the time of diagnosis and during the last cycle of chemotherapy and to evaluate whether chemotherapy may aggravate these patients' sleep disorders.

\section{Patients And Methods}

The study was carried out at the Breast Cancer Center of Chongqing, The First Affiliated Hospital of Chongqing Medical University. We designed a study with 308 women with primary breast cancer, including 194 at the time of initial diagnosis (ID) and 114 before the last cycle of chemotherapy (LC), from January 2018 to October 2018. Chongqing is a quasi-first-tier city in China (with a population of 31.4 million residents). All patients gave written informed consent. This study was approved by the Ethics Committee of the First Affiliated Hospital of Chongqing Medical University.

\section{Methods}

This study comprised assessments at ID and LC. This article focuses on these two assessments to better capture the changes in sleep disorders that may occur between ID and LC. All enrolled patients were assessed for the status and manifestations (such as the mean scores for sleep latency, habitual sleep efficiency, sleep disturbance, and daytime dysfunction) of sleep disorders and the status of the drugs using to help the patients fall sleep as measured via the PSQI. When breast cancer patients were diagnosed in our department, we gave them the PSQI. Similarly, when breast cancer patients underwent the last chemotherapy in our department, we gave them the PSQI. 
The characteristics of the sleep disorders and components of the PSQI were compared between the patients at the time of ID and those at the time of LC using the independent sample T test with SPSS software (version 22.0). When the $P$ value was less than 0.05 , it was considered statistically significant. We mainly analyzed differences in subjective sleep quality, sleep duration, sleep disturbance, habitual sleep efficiency, sleep medications, sleep latency and daytime dysfunction between the two groups.

\section{Results}

The prevalence of sleep disorders in patients at the time of ID with breast cancer was approximately $50 \%$ $(97 / 194)$, which was significantly lower than that $(65.8 \%, 75 / 114)$ in breast cancer patients before the last cycle of chemotherapy ( $\mathrm{p} \otimes 0.05$ ) (Table 1 ). However, both groups of patients rarely used drugs to help them fall sleep ( $P>0.05$ ) (Table 2). The mean scores for sleep latency, habitual sleep efficiency, sleep disturbance, and daytime dysfunction $(1.68,1.08,1.65,1.37)$ in breast cancer patients prior to the last cycle of chemotherapy were obviously worse than those $(1.29,0.71,1.26,1.01)$ in breast cancer patients at the time of initial diagnosis $(p<0.05)$ (Table 2). Correspondingly, the mean scores for sleep latency and habitual sleep efficiency in breast cancer patients prior to the last cycle of chemotherapy $(79 \%, 39.5 \mathrm{~min})$ were obviously worse than those $(84 \%, 29.3 \mathrm{~min})$ in breast cancer patients at the time of ID $(p<0.05)$ (Table 3).

\section{Discussion}

Sleep disorders are the most common symptoms reported by patients with breast cancer. Most studies reported that $30 \%$ to $50 \%$ of newly diagnosed cancer patients have sleep disorders. Although the etiology of sleep disorders comorbid with cancer is most likely multifactorial, the results of the current study suggest that adjuvant cancer treatments are associated with an increased severity of sleep disorders, mediated by side effects of the treatment. To the best of our knowledge, there are few studies about the manifestations of sleep disorders and the status of the drugs used to help Chinese breast cancer patients fall asleep at the time of diagnosis and before the last cycle of chemotherapy measured with the PSQI.

In this study, we analyzed 308 Chinese women with primary breast cancer, including 194 breast cancer patients at ID and 114 breast cancer patients before LC. All enrolled patients were assessed for sleep disorders by completing the PSQI. The prevalence of sleep disorders in breast cancer patients at ID was approximately $50 \%$, which was still significantly lower than that $(65.8 \%)$ in breast cancer patients before LC (Table 1). However, both groups of patients rarely used drugs to help them fall sleep $(p<0.05)$ (Table 2). The mean scores for sleep latency, habitual sleep efficiency, sleep disturbance, and daytime dysfunction were better in breast cancer patients at ID than before LC $(p<0.05)($ Table 2$)$. Symptoms of daytime dysfunction and sleep disturbance are significantly aggravated in Chinese women with primary breast cancer receiving chemotherapy. This means that chemotherapy treatment may lead to women with breast cancer taking a long time to fall asleep, having an increased frequency of using the bathroom, having difficulty breathing, waking up in the middle of the night, coughing, snoring, dreaming, experiencing pain and experiencing temperature-based discomfort. At the same time, in our study, there 
was no significant difference in subjective sleep quality, probably because breast cancer patients have a high prevalence of sleep disorders, and the patients' sleep quality has long been poor.

Studies have shown that sleep disorders may be caused via multiple mechanisms. Particular biomarkers, such as serum levels of cortisol, melatonin, and serotonin, are all associated with sleep disorder symptoms during the chemotherapy cycle. It is possible that differences in sleep disorders could be affected by the cancer itself, cancer treatments, and/or side effects associated with those treatments[1922]. For example, chemotherapy may disrupt the patients' immune function, and some data show that sleep disorders are related to dysregulated immune functioning as measured by increased levels of biomarkers such as interleukin-6 (IL-6), tumor necrosis factor, and C-reactive protein. Chemotherapyinduced inflammation can also affect sleep quality, and the side effects of chemotherapy and the drugs that are used to prevent them can also lead to increased severity of sleep disorders. For instance, using dexamethasone during chemotherapy can cause insomnia[23].

The limitation of this study is that there is no one-to-one correspondence between the patients of Chinese breast cancer at the time of diagnosis and during the last cycle of chemotherapy.

The limitation of this study is that the breast cancer patients which were enrolled are no one-to-one correspondence between the first diagnosis and during the last cycle of chemotherapy. This study does not take into account the fatigue and depression in breast cancer patients, which requires further study.

In summary, the symptoms of sleep disorders were more prevalent and severe in patients with an initial diagnosis of breast cancer than in the general population and were further aggravated in patients during chemotherapy. Chemotherapy mainly affects sleep latency, habitual sleep efficiency, sleep disturbance, and daytime dysfunction in breast cancer patients. Breast cancer patients should be monitored and managed to prevent the aggravation of sleep disorders during chemotherapy. In addition, our study reveals that Chinese breast cancer patients rarely use drugs to intervene sleep disorders. However, it is necessary to use drugs to relieve the symptoms of sleep disorders in breast cancer patients.

\section{Abbreviations}

ID Initial diagnosis;

LC the last cycle of chemotherapy

PSQI the Pittsburgh Sleep Quality Index

\section{Declarations}

\section{Ethics approval and consent to participate}

The study was approved and registered by the local ethics committee of the First Affiliated Hospital of Chongqing Medical University (Reg. No. 2017-044), all patients gave written informed consent. 


\section{Consent for publication}

Not applicable

\section{Availability of data and material}

The datasets used and/or analysed during the current study are available from the corresponding author on reasonable request.

\section{Competing interests}

The authors declare that they have no competing interests.

\section{Funding}

There were no sources of funding for this study.

\section{Authors' contributions}

$\mathrm{BZ} / \mathrm{JL} / \mathrm{HL} / \mathrm{HL} / \mathrm{HC}$ participated in the study design, data collection and drafted the manuscript. $\mathrm{ZX/KW/LK}$ assisted in literature search and provided critical revision of the manuscript for important intellectual content. All authors read and approved the final manuscript.

\section{Contributor Information}

Baoshan Zou E-mail:zoubaoshan@foxmail.com

Hong Li E-mail:525799542@qq.com

Jiashuo Liu E-mail:jiashuoliu@foxmail.com

Zhou Xu E-mail:dr_zhouxu@foxmail.com

Haoran Chen E-mail:Alfred_1993rx@hotmail.com

Hao Li E-mail:Irgnyygmx@163.com

Kainan Wu E-mail:wukainanlaoshi@163.com

Lingquan Kong E-mail:huihuikp@163.com 


\section{References}

1.De Angelis, R., et al., Cancer survival in Europe 1999-2007 by country and age: results of EUROCARE-5 -a population-based study. The Lancet Oncology, 2014. 15(1): p. 23-34.

2.Jemal, A., et al., Global cancer statistics. CA Cancer J Clin, 2011. 61(2): p. 69-90.

3.Bosetti, C., et al., The decline in breast cancer mortality in Europe: an update (to 2009). Breast, 2012. 21(1): p. 77-82.

4.Ferlay, J., et al., Cancer incidence and mortality worldwide: sources, methods and major patterns in GLOBOCAN 2012. Int J Cancer, 2015. 136(5): p. E359-86.

5.Berger, A.M., et al., Values of sleep/wake, activity/rest, circadian rhythms, and fatigue prior to adjuvant breast cancer chemotherapy. J Pain Symptom Manage, 2007. 33(4): p. 398-409.

6.Savard, J., et al., Prevalence, clinical characteristics, and risk factors for insomnia in the context of breast cancer. Sleep, 2001. 24(5): p. 583-90.

7.Savard, J., et al., Insomnia in men treated with radical prostatectomy for prostate cancer. Psychooncology, 2005. 14(2): p. 147-56.

8.Savard, J., et al., Prevalence, natural course, and risk factors of insomnia comorbid with cancer over a 2month period. J Clin Oncol, 2009. 27(31): p. 5233-9.

9.Carpenter, J. S., et al., Sleep, fatigue, and depressive symptoms in breast cancer survivors and matched healthy women experiencing hot flashes. Oncol Nurs Forum, 2004. 31(3): p. 591-5598.

10.Palesh, O. G., et al., Prevalence, demographics, and psychological associations of sleep disruption in patients with cancer: University of Rochester Cancer Center-Community Clinical Oncology Program. J Clin Oncol, 2010. 28(2): p. 292-8.

11.Sanford, S. D., et al., Longitudinal prospective assessment of sleep quality: before, during, and after adjuvant chemotherapy for breast cancer. Support Care Cancer, 2013. 21(4): p. 959-67.

12.Enderlin, C. A., et al., Sleep across chemotherapy treatment: a growing concern for women older than 50 with breast cancer. Oncol Nurs Forum, 2010. 37(4): p. 461-a3.

13.Beck, S. L., et al., Sleep quality after initial chemotherapy for breast cancer. Support Care Cancer, 2010. 18(6): p. 679-89.

14.Savard, J., et al., Cancer treatments and their side effects are associated with aggravation of insomnia: Results of a longitudinal study. Cancer, 2015. 121(10): p. 1703-11. 
15.Chen, M. L., C. T. Yu, and C. H. Yang, Sleep disturbances and quality of life in lung cancer patients undergoing chemotherapy. Lung Cancer, 2008. 62(3): p. 391-400.

16.Van Onselen, C., et al., Trajectories of sleep disturbance and daytime sleepiness in women before and after surgery for breast cancer. J Pain Symptom Manage, 2013. 45(2): p. 244-60.

17.Ho, R. T. and T. C. Fong, Factor structure of the Chinese version of the Pittsburgh sleep quality index in breast cancer patients. Sleep Med, 2014. 15(5): p. 565-9.

18.Buysse, D. J., et al., The Pittsburgh Sleep Quality Index: a new instrument for psychiatric practice and research. Psychiatry Res, 1989. 28(2): p. 193-213.

19.Lee, B. N., et al., A cytokine-based neuroimmunologic mechanism of cancer-related symptoms. Neuroimmunomodulation, 2004. 11(5): p. 279-92.

20.Mills, P. J., et al., The effects of standard anthracycline-based chemotherapy on soluble ICAM-1 and vascular endothelial growth factor levels in breast cancer. Clin Cancer Res, 2004. 10(15): p. 4998-5003.

21.Payne, J., et al., Biomarkers, fatigue, sleep, and depressive symptoms in women with breast cancer: a pilot study. Oncol Nurs Forum, 2006. 33(4): p. 775-83.

22.Wood, L. J., et al., Cancer chemotherapy-related symptoms: evidence to suggest a role for proinflammatory cytokines. Oncol Nurs Forum, 2006. 33(3): p. 535-42.

23.Niiranen, A. and K. Mattson, Antiemetic efficacy of nabilone and dexamethasone: a randomized study of patients with lung cancer receiving chemotherapy. Am J Clin Oncol, 1987. 10(4): p. 325-9.

\section{Tables}

Table 1. Different scores in two groups according to PSQI

\begin{tabular}{llll}
\hline & ID & LC & $P$ Value \\
\hline $0-5$ & $97(50.0)$ & $39(34.2)$ & \\
$6-10$ & $70(36.1)$ & $44(38.6)$ & \\
$11-15$ & $24(12.4)$ & $25(21.9)$ & \\
& & & \\
$16-21$ & $3(1.5)$ & $6(5.3)$ & \\
$6-21$ & $97(50.0)$ & $75(65.8)$ & 0.007 \\
& & & \\
\hline
\end{tabular}

ID: Initial diagnosis ( $n=194) ;$ LC: the cycle of last chemotherapy $(n=114)$; frequency (percent); PSQI: the Pittsburgh Sleep Quality Index. 
Table 2. Principal component analysis of the Pittsburgh Sleep Quality Index (PSQI) at initial diagnosis and before the cycle of last chemotherapy

\begin{tabular}{lccl}
\hline Component & ID & LC & $P$ value \\
\cline { 2 - 3 } & \multicolumn{2}{c}{ Mean \pm SD } & \\
\hline Subjective sleep quality & 1.15 & 1.32 & 0.069 \\
Sleep latency & 1.29 & 1.68 & $0.001^{+}$ \\
Sleep duration & 0.59 & 0.78 & 0.099 \\
Habitual sleep efficiency & 0.71 & 1.08 & $0.03^{+}$ \\
Sleep disturbances & 1.26 & 1.65 & $00.001^{+}$ \\
Daytime dysfunction & 1.01 & 1.37 & $0.04^{+}$ \\
Sleep medications & 0.07 & 0.08 & 0.81 \\
\hline
\end{tabular}

ID: Initial diagnosis ( $n=194)$; LC: the cycle of last chemotherapy $(n=114) ;$ PSQI: the Pittsburgh Sleep Quality Index."+": All correlations are statistically significant $(P<0.05)$.

Table 3. Actual value at initial diagnosis and before the cycle of last chemotherapy

\begin{tabular}{lccc}
\hline \multicolumn{1}{c}{ Index } & \multicolumn{2}{c}{ ID } & LC \\
\cline { 2 - 3 } & \multicolumn{3}{c}{ Mean \pm SD } \\
\cline { 2 - 3 } \\
\cline { 2 - 3 } & $49.2 \pm 9.8$ & $48.5 \pm 9.3$ & 00.05 \\
Sleep latency(min) & $29.3 \pm 27.1$ & $39.5 \pm 34.2$ & 0.007 \\
Habitual sleep efficiency & $84 \% \pm 16 \%$ & $79 \% \pm 17 \%$ & 0.013 \\
Sleep duration(h) & $7.0 \pm 1.5$ & $6.9 \pm 1.7$ & 0.067 \\
\hline
\end{tabular}

All data are presented as mean $\pm S D$. 